\title{
Minimalinvasive Chirurgie bei Malignomen des Gastrointestinaltrakts: Kolon - Pro-Position
}

\author{
Christoph Späth Tara Müller Ulrich Nitsche Matthias Maak Samuel A. Käser \\ Jörg Kleeff Franz G. Bader
}

Chirurgische Klinik und Poliklinik, Klinikum rechts der Isar, Technische Universität München, Deutschland

\section{Schlüsselwörter}

Kolonkarzinom · Chirurgie, laparoskopische ·

Chirurgie, minimalinvasive

\section{Zusammenfassung}

Hintergrund: Laparoskopische Resektionen von Kolonkarzinomen werden in Deutschland immer häufiger durchgeführt. Dabei müssen sich die minimalinvasiven Verfahren an den onkologischen Ergebnissen der offenen Chirurgie messen, die bisher den Goldstandard darstellen. Die vorliegende Arbeit soll anhand der evidenzbasierten Literatur einen kritischen Überblick über die Kurz- und Langzeitergebnisse der laparoskopischen Kolonchirurgie geben und sie in Bezug zu den Ergebnissen der offenen Chirurgie setzen. Methoden: Die Grundlage dieser Übersichtsarbeit bilden die fünf größten prospektiv randomisierten Studien zum Vergleich laparoskopischer und offener Chirurgie beim Kolonkarzinom (ALCCaS, Barcelona Trial, CLASICC Trial, COLOR Study und COST Study) sowie die bisher größte und aktuellste Metaanalyse. Im Folgenden sollen die erwähnten Studien hinsichtlich der Kurzzeit- und Langzeitergebnisse kritisch zusammengefasst sowie die wichtigsten Endpunkte beleuchtet werden. Ergebnisse: Die laparoskopische Chirurgie des Kolonkarzinoms ist hinsichtlich der onkologischen Langzeitergebnisse der offenen Resektion zumindest gleichwertig. Vorteile bestehen in der frühen postoperativen Phase sowie im perioperativen Outcome. Die höheren direkten Kosten werden durch kürzere Liegezeiten, niedrigere Morbidität und weniger Re-Interventionen bezüglich Adhäsionen und Narbenhernien ausgeglichen. Schlussfolgerungen: Eine kritische und individuelle Indikationsstellung vorausgesetzt, sollte bei entsprechender Expertise und Erfahrung den laparoskopischen Verfahren zur Therapie des Kolonkarzinoms der Vorzug gegeben werden, um den Patienten bei gleichwertigen onkologischen Langzeitergebnissen die Vorteile der minimalinvasiven Chirurgie nicht vorzuenthalten.

\section{KARGER \\ Fax +497614520714}

Information@Karger.com

www.karger.com (c) 2013 S. Karger GmbH, Freiburg

$1662-6664 / 13 / 0296-0382 \$ 38.00 / 0$

Accessible online at:

www.karger.com/vim
Keywords

Colon cancer - Laparoscopic surgery . Minimally invasive surgery

\section{Summary}

Minimally Invasive Surgery for Malignancies of the Gastrointestinal Tract: Colon - Pro Position

Background: Laparoscopic resections for colon cancer are increasingly performed in Germany. The results of the laparoscopic procedures have to compete with the excellent oncological outcomes of standard open resections. This article gives an overview on the short- and long-term results of laparoscopic colon cancer surgery compared to open resection, referring to evidence-based literature. Methods: The article reviews the five largest prospective randomized trials comparing laparoscopic and open resection for colon cancer (ALCCaS, Barcelona Trial, CLASICC Trial, COLOR Study, and COST Study) as well as the largest and most recent meta-analysis. Shortand long-term results are summarized and the most important endpoints are presented. Results: The oncologic long-term results of laparoscopic surgery are at least equal to those of standard open surgery. There are advantages within the early postoperative period and for the perioperative outcome. The higher direct costs of laparoscopic resections are equalized by shorter hospital stays, less morbidity, and less re-interventions regarding incisional hernia and intra-abdominal adhesions. Conclusions: Given a critical and individualized indication, laparoscopic surgery for colon cancer performed by an experienced and well-trained surgeon should be the treatment of choice, enabling patients to benefit from earlier functional recovery with no detriment to longterm oncological outcomes. 


\section{Einleitung}

Darmkrebs stellt in Deutschland die zweithäufigste Krebserkrankung nach dem Mammakarzinom (Frauen) und dem Prostatakarzinom (Männer) dar. Im Jahr 2012 verzeichnete das Robert Koch-Institut etwa 69000 Neuerkrankungen, wobei Männer mit 38000 Fällen etwas häufiger betroffen waren als Frauen mit 31000 Fällen (Zentrum für Krebsregisterdaten, Robert Koch-Institut).

Der Goldstandard zur Therapie des Kolonkarzinoms im nicht metastasierten Stadium ist immer noch die primäre Resektion in offener Technik. Die Ergebnisse der laparoskopischen Verfahren müssen sich hier einem kritischen Vergleich unterziehen und sich mit denen der offenen Chirurgie messen, insbesondere unter Berücksichtigung des von Hohenberger et al. [1] propagierten Konzepts der kompletten mesokolischen Exzision (CME). Durch das Konzept der CME, das letztlich die logische und folgerichtige Konsequenz aus den Erfahrungen mit der totalen mesorektalen Exzision (TME) beim Rektumkarzinom darstellt, kann eine hohe Standardisierung der Chirurgie des Kolonkarzinoms mit exzellenten onkologischen Ergebnissen erreicht werden [2].

Im Jahr 1991 wurde erstmalig eine laparoskopisch-onkologische Sigmaresektion durchgeführt [3] und steht seitdem in Konkurrenz zur konventionellen offenen Technik mittels medianer Laparotomie.

Die Anzahl der laparoskopischen Resektionen beim Kolonkarzinom steigt dabei stetig an, von 4\% im Jahr 2004 auf 22\% im Jahr 2011 (DeStatis, OPS-Statistik 2011). Damit liegt Deutschland im internationalen Vergleich aber immer noch deutlich hinter anderen Nationen zurück. So werden in Südkorea $74 \%$ aller Kolonkarzinome minimalinvasiv reseziert, gefolgt von den USA mit 41\% und Spanien mit 35\% (World Health Statistics 2011, Weltgesundheitsorganisation (WHO)). Die Entwicklung zeigt aber, dass auch in Deutschland minimalinvasive Verfahren immer öfter der offenen Chirurgie vorgezogen werden. Deshalb sollte weniger die Frage sein «laparoskopisch oder nicht», sondern eher «onkologisch adäquat oder nicht».

In der evidenzbasierten Medizin sind wenige Fragestellungen wissenschaftlich so nachhaltig untersucht wie der Vergleich zwischen laparoskopischer und offener Chirurgie beim Kolonbzw. Rektumkarzinom. Mittlerweile sind über 5000 Patienten in prospektiv randomisierte Studien eingeschlossen, die mit robusten Langzeitdaten den Stellenwert der minimalinvasiven Verfahren untermauern. Die vorliegende Arbeit hat zum Ziel, einen Überblick, aber auch eine kritische Bewertung der wichtigsten Studien in diesem Zusammenhang darzulegen.

\section{Vorgehensweise}

Die Grundlage der vorliegenden Arbeit sollen die fünf größten prospektiv randomisierten Studien aus Europa, Aust- ralien und den USA darstellen, die sich dem Vergleich von laparoskopischer und offener Chirurgie beim Kolonkarzinom widmen: Australasian Laparoscopic Colon Cancer Surgical Trial (ALCCaS) [4, 5], Barcelona Trial [6, 7], Conventional Versus Laparoscopic-Assisted Surgery in Colorectal Cancer (CLASICC) Trial [8-10], Colon Carcinoma Laparoscopic or Open Resection (COLOR) Study [11] und Clinical Outcomes of Surgical Therapy (COST) Study [12]. Darüber hinaus liegt eine kürzlich publizierte Metaanalyse vor, die sich ebenfalls kritisch mit dem Vergleich zwischen offener und laparoskopischer Chirurgie beim Kolonkarzinom auseinandersetzt [13].

Im Folgenden sollen die erwähnten Studien hinsichtlich der Kurz- sowie der Langzeitergebnisse kritisch zusammengefasst und die wichtigsten Endpunkte beleuchtet werden.

\section{Ergebnisse}

\section{Short-Term Outcomes}

\section{Konversionsrate}

In der multizentrisch durchgeführten ALCCaS-Studie wurden zwischen 1998 und 2005 insgesamt 601 Patienten eingeschlossen. Insgesamt 299 Patienten wurden in den laparoskopischen Arm randomisiert, 302 Patienten wurden dem Arm für die offene Resektion zugeteilt. Die Konversionsrate betrug $14,6 \%$ [4].

Die Barcelona-Studie wurde in den Jahren 1993-1998 in der Universität von Barcelona durchgeführt; dabei wurden 111 Patienten laparoskopisch und 108 offen operiert (insgesamt 219). Die Rate an Konversionen betrug 11\% [6].

An der europäischen CLASICC-Studie nahmen im Zeitraum von 1996 bis 2002 insgesamt 27 Zentren teil. Es konnten 794 Patienten eingeschlossen werden, 526 in den laparoskopischen und 268 in den offenen Arm. Bei 25\% der Patienten war eine Konversion notwendig [8].

Von 1997 bis 2003 wurde die internationale COLOR-Studie unter Beteiligung von 29 Zentren durchgeführt. Insgesamt wurden 1076 Patienten eingeschlossen, von denen 534 laparoskopisch operiert wurden. In dieser Studie lag die Konversionsrate bei $19 \%$ [11].

Die COST-Studie wurde von 1994 bis 2001 durchgeführt. In 48 Zentren wurden 863 Patienten aufgrund eines Kolonkarzinoms operiert, 435 in laparoskopischer und 428 in offener Technik. Die Rate an Konversionen lag in dieser Studie bei $21 \%$ [12].

Zusammengefasst wurden in den fünf Studien 1620 Operationen laparoskopisch begonnen. Bei 308 Fällen musste eine Konversion auf ein offenes Verfahren erfolgen (19,0\%).

Die Metaanalyse von Ohtani et al. [13] fasst zwölf randomisiert-kontrollierte Studien mit insgesamt 4614 Patienten zusammen. Bei 2444 wurde eine laparoskopische Resektion durchgeführt, 2170 wurden offen operiert. Die Konversionsrate wurde mit einer Rate von 3-46,4\% angegeben. 


\section{Operationszeit}

In der ALCCaS-Studie wurde eine durchschnittliche Operationszeit von $170 \mathrm{~min}$ bei laparoskopischen im Vergleich zu 115 min bei offenen Operationen beobachtet $(p<0,001)$ [4]. Auch in der Barcelona-Studie dauerte die laparoskopische Resektion mit durchschnittlich 142 min signifikant länger (vs. 118 min; $p$ < 0,001) [6]. In der CLASICC-Studie war die Zeit bei laparoskopischen Eingriffen mit im Schnitt 180 min ebenfalls deutlich länger als bei der offenen Resektion [8]. Die COLOR-Studie zeigte mit 202 versus 170 min ähnliche Ergebnisse $(p<0,001)$ [11]. Die Ergebnisse der Metaanalyse zeigen, dass die laparoskopische Resektion im Schnitt 42 min länger dauert als die offene Operation $(p<0,00001)$ [13].

\section{Intraoperativer Blutverlust}

Beim intraoperativen Blutverlust wurden in der ALCCaSStudie keine signifikanten Unterschiede zwischen den einzelnen Gruppen gesehen (beide im Mittel 100 ml, p =0,17). Auch die Anzahl der Patienten, die eine Bluttransfusion benötigten, war nicht signifikant unterschiedlich [4]. In der Barcelona-Studie war der durchschnittliche Blutverlust mit $105 \mathrm{ml}$ im laparoskopischen Arm signifikant geringer (vs. $193 \mathrm{ml}$; p < 0,001) [6]. Ähnliche Ergebnisse zeigte die COLOR-Studie mit durchschnittlich $100 \mathrm{ml}$ Blutverlust bei laparoskopischen versus 175 $\mathrm{ml}$ bei offenen Resektionen ( $\mathrm{p}<0,0001)$ [11]. In der CLASICCStudie war der intraoperative Blutverlust im offenen Arm signifikant höher $(\mathrm{p}=0,002)$ [8]. Auch die Metaanalyse zeigte einen signifikant reduzierten Blutverlust für das laparoskopische Vorgehen (mittlere Abweichung vom Median (MD) $=-103,90$, $95 \%$-Konfidenzintervall $(\mathrm{CI})=-180,88,-26,91 ; \mathrm{p}<0,0001)$ [13].

\section{Kostaufbau und postoperativer Ileus}

Die ALCCaS-Studie zeigte keine signifikanten Unterschiede beim Auftreten von prolongiertem postoperativem Ileus über 6 Tage (5,1 vs. 6,7\%; $p=0,487)$ [4]. Die Metaanalyse hingegen zeigte bei laparoskopisch operierten Patienten eine deutlich niedrigere Rate an postoperativem Ileus (Odds Ratio $(\mathrm{OR})=0,40,95 \% \mathrm{CI}=0,25,0,66 ; \mathrm{p}=0,0003)$ [13].

Die laparoskopisch operierten Patienten der BarcelonaStudie konnten im Schnitt nach 54 h mit vollwertiger Kost beginnen und somit signifikant früher als der Vergleichsarm mit $85 \mathrm{~h}(\mathrm{p}<0,001)$ [6]. Auch die orale Flüssigkeitsaufnahme war im laparoskopischen Arm sowohl in der ALCCaS-Studie (2,4 vs. 3,0 Tage; $\mathrm{p}<0,001)$ als auch in der COLOR-Studie (2,9 vs. $3,8$ Tage; $p<0,001)$ früher möglich $[4,11]$. Die Zeit bis zum vollständigen Kostaufbau war in der Metaanalyse für die laparoskopische Gruppe signifikant kürzer $(\mathrm{MD}=-0,81,95 \%$ $\mathrm{CI}=-1,03,-0,06 ; \mathrm{p}<0,0001)[13]$.

\section{Dauer des Krankenhausaufenthaltes}

Im Mittel war der Krankenhausaufenthalt für beide Gruppen in der ALCCaS-Studie nicht unterschiedlich (9,5 vs. 10,6 Tage; $p=0,068$ ) [4]. In den anderen vier Studien war der Krankenhausaufenthalt in der laparoskopischen Gruppe al- lerdings signifikant kürzer $[6,8,11,12]$. Auch die Metaanalyse bestätigt diese Ergebnisse; die Patienten waren 2,2 Tage weniger hospitalisiert $(p<0,00001)$ [13].

Patienten, bei denen eine Konversion vom laparoskopischen auf das offene Verfahren erfolgte, lagen in der ALCCaS-Studie signifikant länger im Krankenhaus (14,6 vs. 8,6 Tage; $\mathrm{p}<0,0001)$ [4].

\section{Morbidität und Mortalität}

In der ALCCaS-Studie gab es keinen Unterschied im Auftreten von postoperativen Komplikationen in beiden Gruppen. Die Rate an Re-Operationen (5,4 vs. 4,4\%; p = 0,543) und die Rate an Anastomoseninsuffizienzen (1,4 vs. 3,4\%; $\mathrm{p}=$ 0,110) war ebenfalls nicht unterschiedlich [4]. Die BarcelonaStudie zeigte dagegen Vorteile für das laparoskopische Verfahren. Hier war die Gesamtzahl der Komplikationen deutlich geringer $(10,8$ vs. $28,7 \%$; $p<0,001)$ [6]. Die CLASICCStudie, die COLOR-Studie und die COST-Studie wiederum konnten keinen signifikanten Vorteil für eine der beiden Gruppen zeigen $[8,11,12]$. In der Metaanalyse zeigte sich ein signifikanter Vorteil für das laparoskopische Verfahren (OR $=0,73,95 \%$ CI 0,56, 0,95; $\mathrm{p}=0,02)$ [13].

Bei der perioperativen Mortalität gab es in der Zusammenschau der Studien keine Unterschiede zwischen den Vergleichsgruppen (ALCCaS 1,4 vs. 0,7\%, p =0,448; Barcelona 0,9 vs. $2 \%$; COLOR 1 vs. $2 \%, p=0,45$; COST 0,4 vs. $0,9 \%, p$ $=0,4)$. Auch die Metaanalyse konnte keinen Vorteil für eine Methode zeigen $(\mathrm{OR}=0,72,95 \% \mathrm{CI}=0,35,1,48)$ [13].

\section{Anzahl der resezierten Lymphknoten}

Im Mittel wurden in der ALCCaS-Studie in beiden Gruppen 13 Lymphknoten reseziert $(p=0,145)$ [4]. In der Barcelona-Studie war die Lymphknotenausbeute in beiden Gruppen mit im Schnitt 11,1 Lymphknoten ebenfalls gleich [6]. Auch in der Metaanalyse wurde kein Unterschied bei der Anzahl der entfernten Lymphknoten beobachtet $(\mathrm{MD}=-0,48$, $95 \% \mathrm{CI}=-1,27,0,31 ; \mathrm{p}=0,23)[13]$.

\section{Kosten}

Die Metaanalyse zeigte keinen signifikanten Unterschied hinsichtlich der Kosten. In die Analyse gingen jedoch nur zwei Studien ein, wobei sich in der CLASICC-Studie kein Kostenunterschied bezüglich der beiden Verfahren zeigte, die Arbeit von Ohtani et al. [13] jedoch signifikant geringere Kosten für das offene Vorgehen berichtete. Das deutsche Gesundheitssystem zugrundegelegt, sind für die laparoskopische Resektion deutlich höhere Kosten zu erwarten.

\section{Long-Term Outcomes}

\section{Lokalrezidive und distante Metastasierung}

Die ALCCaS-Studie überblickt ein mittleres Follow-up von 5,2 Jahren mit insgesamt 3023 Patientenjahren. Es gab keinen 
Unterschied in der Gesamtrezidivrate. In der laparoskopischen Gruppe kam es bei 13,7\% der Patienten zu einem Rezidiv, in der offenen Gruppe bei 14,8\% ( $p=0,73)$, wobei nicht nach Art des Rezidivs unterschieden wurde. Dabei war die Verteilung der Tumorstadien in beiden Gruppen gleich [5].

In der Barcelona-Studie beträgt das mittlere Follow-up 7,9 Jahre. In der Laparoskopie-Gruppe kam es in $18 \%$ der Fälle zu einem Rezidiv (lokal und distant), in der Gruppe mit offenen Resektionen zeigten 28\% der Patienten ein Rezidiv, dies jedoch, ohne statistische Signifikanz zu erreichen $(p=0,07)$ [7].

In der CLASICC-Studie wurden die Patienten im Mittel 5,2 Jahre nachbeobachtet. Hier gab es sowohl bei der Rate an Lokalrezidiven als auch bei der Rate an Fernmetastasen keinen Unterschied zwischen beiden Gruppen. Bei den Wundrezidiven bzw. Rezidiven im Bereich der Trokarnarben zeigte sich ein signifikanter Unterschied. Bei 2 Patienten $(0,3 \%)$ zeigte sich eine Metastase im Bereich der Laparotomienarbe, bei 10 Patienten $(1,8 \%)$ trat ein Rezidiv im Bereich der ehemaligen Trokarkanäle auf $(p=0,002)$ [10].

Die COST-Studie überblickt ein mittleres Follow-up von 4,4 Jahren. Insgesamt 76 Patienten $(17,4 \%)$ aus der laparoskopischen Gruppe und 84 Patienten aus der offenen Gruppe $(19,6 \%)$ entwickelten ein Rezidiv. In der tumorbezogenen Auswertung zeigte sich ebenfalls kein signifikanter Unterschied in der Rate an Rezidiven. Die Rate der Wundrezidive war nicht signifikant unterschiedlich (0,4 vs. $0,2 \% ; p=0,50)$ [12].

In der Metaanalyse von Ohtani et al. [13] war weder das Auftreten von Lokalrezidiven $(\mathrm{OR}=0,82,95 \% \mathrm{CI}=0,51$, 1,31 ) noch das Auftreten von Fernmetastasen ( $\mathrm{OR}=0,97$, $95 \% \mathrm{CI}=0,78,1,21)$ signifikant unterschiedlich. Dies gilt auch für die Gesamtrate an Rezidiven ( $\mathrm{OR}=0,96,95 \% \mathrm{CI}=0,81$, 1,15). Die Rate an Wundrezidiven zeigte einen Trend hinsichtlich der offenen Gruppe, ohne statistische Signifikanz zu erreichen $(\mathrm{OR}=2,40,95 \% \mathrm{CI}=0,87,6,61 ; \mathrm{p}=0,87)$.

\section{Überleben}

Für das 5-Jahres-Überleben konnte in der ALCCaS-Studie kein Unterschied zwischen beiden Gruppen nachgewiesen werden (77,7 vs. 76,0\%: Log-Rank P =0,940). Auch im krankheitsfreien Überleben nach 5 Jahren gab es keinen Unterschied (72,3 vs. 71,7\%; Log-Rank P = 0,823) [5].

In der Barcelona-Studie zeigte sich ein Trend zu längerem krankheitsfreien Überleben im Laparoskopie-Arm. Am Ende des Beobachtungszeitraums wurden $84 \%$ der Patienten ohne Rezidiv in der laparoskopischen Gruppe registriert, in der offenen Gruppe waren es 73\%. Dieser Unterschied erreichte keine statistische Signifikanz ( $p=0,07)$ [7].

Das krankheitsfreie Überleben in der CLASICC-Studie zeigte keine Unterschiede zwischen beiden Gruppen. Das mediane krankheitsfreie Überleben in der laparoskopischen Gruppe lag bei 89,5 Monaten, in der Vergleichsgruppe waren es 77,0 Monate (Log-Rank P = 0,589) [10].

Das Gesamtüberleben (Hazard Ratio $(\mathrm{HR})=0,91,95 \% \mathrm{CI}$ $=0,68,1,21 ; \mathrm{p}=0,51)$ wie auch das krankheitsfreie Überleben
$(\mathrm{HR}=0,95,95 \% \mathrm{CI}=0,74,1,23 ; \mathrm{p}=0,70)$ waren in beiden Gruppen der COST-Studie nicht unterschiedlich. Auch eine Subgruppenanalyse nach Tumorstadien zeigte keinen Unterschied im Überleben [12].

Bezüglich krebsbedingter Sterblichkeit gab es in der Metaanalyse keinen Vorteil für eine der beiden Gruppen (OR = $0,79,95 \% \mathrm{CI}=0,55,1,11)$. Ebenso war die Gesamtmortalität in beiden Gruppen ähnlich $(\mathrm{OR}=0,90,95 \% \mathrm{CI}=0,70,1,15)[13]$.

\section{Überleben nach Konversion}

In der Subgruppe der Patienten, bei denen eine Konversion von laparoskopisch auf offen stattfand, zeigte sich in der ALCCaS-Studie ein signifikant schlechteres krankheitsfreies 5-Jahres-Überleben im Vergleich zu den laparoskopisch operierten Patienten (55,7 vs. 76,0\%; Log-Rank $\mathrm{P}<0,001$ ) [5]. Die 3- und 5-Jahres-Analyse der CLASICC-Studie zeigte ein statistisch signifikant schlechteres onkologisches Outcome nach Konversion im Bezug auf das Gesamtüberleben (Kolonund Rektumkarzinome) [9, 14]. Die 10-Jahres-Analyse der CLASICC-Studie hingegen zeigte ein signifikant schlechteres Überleben der konvertierten Patienten mit Kolonkarzinomen sowohl in Bezug auf das krankheitsfreie Überleben als auch auf das Gesamtüberleben [10].

\section{Diskussion}

In der evidenzbasierten Medizin sind wohl nur wenige Fragestellungen mit so robusten Daten hinterlegt wie der Vergleich zwischen offener und laparoskopischer Chirurgie beim Kolon- bzw. Rektumkarzinom. Die fünf größten prospektiv randomisierten Studien allein steuern hier 3553 Patienten zu den weit über 5000 Patienten insgesamt bei [13]. Die letztlich allen Studien und Metaanalysen gemeinsame Konklusion ist die onkologische Gleichwertigkeit des laparoskopischen Vorgehens gegenüber der offenen Technik mit jedoch deutlichen Vorteilen zugunsten der Laparoskopie in der frühen postoperativen Phase [4, 6, 8, 11-13].

Bezüglich der unmittelbar intra- und postoperativen Phase zeigen sich deutlich längere Operationszeiten für das laparoskopische Vorgehen, jedoch auch ein signifikant geringerer Blutverlust [4, 6, 8, 11, 13]. Als Erklärung hierfür werden unter anderem die durch die High-Definition(HD)-Technologie bzw. optische Vergrößerung mögliche präzisere Dissektionstechnik in Kombination mit modernen Dissektionsinstrumenten angeführt [13].

Die technische Gleichwertigkeit des laparoskopischen Vorgehens im Hinblick auf onkologische Standards wird offenbar durch die Daten zu resezierten Lymphknoten bzw. zu positiven zirkumferenziellen Resektionsrändern gezeigt [4, 6, 13]. Hier besteht zwischen beiden Verfahren kein signifikanter Unterschied. Was bis zuletzt jedoch gänzlich fehlt, sind Daten zur histopathologischen Qualitätskontrolle der Resektate der offenen im Vergleich zur laparoskopischen Technik. Wünschens- 
wert wäre hier - analog zur M.E.R.C.U.R.Y.-Klassifikation beim Rektumkarzinom - eine qualitätskontrollierte Studie hinsichtlich der CME-Qualität des laparoskopischen im Vergleich zum offenen Vorgehen beim Kolonkarzinom [15].

Die vorliegenden Daten zeigen weiter einen signifikant schnelleren Kostaufbau bzw. reduzierte postoperative Atoniephasen zugunsten der laparoskopisch operierten Patienten, was in eine schnellere Erholung und kürzere Krankenhausverweildauer mündet [6, 8, 11-13]. Darüber hinaus kann die postoperative Morbidität hinsichtlich septischer Wundkomplikationen und adversen kardiopulmonalen Ereignissen gesenkt werden [8]. Dies ist insbesondere vor dem Hintergrund einer möglicherweise indizierten adjuvanten Chemotherapie von Bedeutung, die nicht selten durch postoperative Komplikationen verspätet beginnt oder unter Umständen gänzlich wegfallen muss.

Das minimalinvasive Vorgehen scheint durch die Reduktion des operativen Traumas an der Bauchdecke bzw. an intraabdominalen Strukturen zu einer signifikanten Reduktion von Narbenhernie bzw. intraabdominellen Adhäsionen zu führen. So zeigt eine kürzlich publizierte Arbeit von Burns et al. [16] die Reduktion von klinisch relevanten Adhäsionen bzw. Narbenhernien bei Patienten nach laparoskopischen kolorektalen Resektionen im Vergleich zum offenen Vorgehen. Dies ist insofern von Bedeutung, als zwar die direkten Kosten des minimalinvasiven Vorgehens höher sind (längere Operationszeit, hoher technischer Aufwand), sich jedoch die Reduktion der Folgekosten (Liegezeit, Re-Interventionsrate bezüglich Adhäsionen/Narbenhernien) als volkswirtschaftlich vorteilhaft erweisen könnte.

In der Chirurgie des Kolonkarzinoms sind aus unserer Sicht - bei allen Vorteilen zugunsten des laparoskopischen Vorgehens in der postoperativen Phase - die onkologischen Ergebnisse das entscheidende Qualitätskriterium. Aber auch hier zeigen die vorliegenden Daten die Gleichwertigkeit der laparoskopischen Verfahren hinsichtlich Gesamtüberleben und krankheitsfreiem Überleben sowie hinsichtlich der Raten an lokaler bzw. distanter Metastasierung [5, 7, 10, 12]. Auch die zu Beginn der laparoskopischen Chirurgie geäußerten Bedenken bezüglich Trokarkanalmetastasen bzw. peritonealer Tumorzelldissemination können widerlegt werden [10].

Ein in diesem Zusammenhang weiterhin kontrovers diskutiertes Phänomen sind die möglicherweise schlechteren onkologischen Ergebnisse nach Konversion von laparoskopischem zu offenem Vorgehen [5, 9, 14].

Einige Studien hierzu zeigen keinen Unterschied im Outcome nach Konversion [17, 18]. Im Gegensatz hierzu bestätigen die Daten der 3- und 5-Jahres-Analyse der CLASICCStudie ein schlechteres Outcome nach Konversion, was sich jedoch statistisch «lediglich» im Gesamtüberleben signifikant unterschiedlich zeigte [9, 14]. Die 10-Jahres-Daten der CLASICC-Studie zeigten jedoch ein signifikant schlechteres krankheitsfreies Überleben und Gesamtüberleben nach Konversion zum offenen Vorgehen bei Kolonkarzinomen. Da sich dies nicht nur im Gesamtüberleben, sondern insbesondere auch im krankheitsfreien Überleben widerspiegelt, machen die Autoren eher den Krankheitsprozess selbst bzw. die Tumorbiologie an sich für das schlechtere Outcome verantwortlich als die Konversion per se. Überaschenderweise beeinflusste die Konversion beim Rektumkarzinom weder das krankheitsfreie Überleben noch das Gesamtüberleben in der Analyse der 10-Jahres-Daten der CLASICC-Studie. Die Gründe hierfür bleiben weiterhin unklar [10].

Die Konversionsrate lag in den dargestellten fünf größten randomisierten kontrollierten Studien zwischen 11 und 25\%, im Mittel bei 19\% [4, 6, 8, 11, 12]. Diese aus heutiger Sicht doch sehr hohe Rate an Konversionen wirft zwei prinzipielle Fragen auf:

- Wie ist die Konversion zu definieren?

- Wie ist die Qualität der laparoskopischen Chirurgie in den zumeist als Multicenter-Studien durchgeführten Untersuchungen zu bewerten?

Vor dem Hintergrund der publizierten Daten, die als Hauptgrund für Konversion ein fortgeschrittenes Tumorstadium identifizieren, muss kritisch hinterfragt werden, ob der Zeitpunkt der Konversion aus onkologischen Gesichtspunkten adäquat gewählt war. Es scheint aus unserer Sicht unstrittig, dass etwa eine Konversion nach Tumoreröffnung unweigerlich zu schlechteren Ergebnissen führen muss. Ist diese Situation aber vergleichbar mit einer Konversion, in der sich der Chirurg nach diagnostischer Laparoskopie und gegebenenfalls limitierter Mobilisation aufgrund seiner Erfahrung und Expertise zu offenem Vorgehen entschließt? Und wie ist in diesem Zusammenhang «Erfahrung und Expertise» zu definieren?

In einigen der vorliegenden Studien mussten sich Chirurgen vor Teilnahme an der Studie einem Trainingsprogramm unterziehen, in dem sie zwischen 20 und 30 laparoskopische Resektionen unter Supervision durchführten, um so einen gewissen Qualitätsstandard innerhalb der Studie zu gewährleisten [8]. Daten von Marusch et al. [19] zeigen jedoch deutlich, dass die Lernkurve für laparoskopische Eingriffe weit über den vorgegebenen 20-30 Resektionen liegt. Darüber hinaus liegen die Konversionsraten an spezialisierten Zentren mit 4-8\% weit unter den in den vorliegenden Arbeiten publizierten Raten [20-22]. Somit sollte die Qualität der laparoskopischen Chirurgie in den einzelnen Studien zumindest kritisch beleuchtet werden, obgleich sie wohl die praktische Realität in den 1990er-Jahren darstellten, in denen die meisten Patienten der betrachteten Studien operiert wurden.

Deshalb muss aus unserer Sicht die Konversion per se nicht zu schlechteren onkologischen Ergebnissen führen, da sich der in den laparoskopischen Techniken Erfahrene durch eine frühzeitige, ja rechtzeitige Konversion auszeichnet und so ein Kompromittieren des onkologischen Ergebnisses verhindern kann.

Trotzdem müssen die potenziell schlechteren Ergebnisse nach Konversion kritisch bewertet werden und dürfen nicht als lediglich statistisches Phänomen vernachlässigt werden. 
Das letzte Jahrzehnt konnte hinsichtlich der technischen Möglichkeiten in der Laparoskopie eine enorme Entwicklung verzeichnen. Darüber hinaus sind, unterstützt durch die Fachgesellschaften in Deutschland, entsprechende Trainingsprogramme inauguriert worden, wenn sie auch noch deutlich hinter den Bemühungen unserer europäischen Nachbarn, insbesondere derjenigen Großbritanniens, zurückliegen. So ist es nicht verwunderlich, dass in Deutschland lediglich etwa $25 \%$ aller kolorektalen Resektionen beim Karzinom in laparoskopischer Technik durchgeführt werden. Schließt man auch die Tatsache, dass die meisten Patienten in den vorliegenden Studien in den 1990er-Jahren operiert wurden, in die Überlegungen mit ein, darf angenommen werden, dass die aktuelle Qualität der laparoskopischen Chirurgie deutlich zugenommen hat.

Trotzdem ist und bleibt die kritische und besonnene Indikationsstellung wohl der entscheidende Faktor. Nicht jeder Tumor und insbesondere nicht jeder Patient ist für ein laparoskopisches Vorgehen geeignet. Auch spielt insbesondere die Tumorlokalisation eine entscheidende Rolle, da z.B. die Flexurenkarzinome mit den konsekutiv notwendigen erweiterten Resektionen hohe Expertise verlangen und so erfahrenen Zentren vorbehalten sind bzw. in offener Technik durchge- führt werden sollten. Darüber hinaus sind die Karzinome des rechten Kolons unter Berücksichtigung des Konzepts der CME technisch weit anspruchsvoller als die des linksseitigen Kolons, insbesondere aufgrund der anatomischen Beziehungen der gastroepiploischen Arkade bzw. des Henle'schen Trunkus zum Pankreaskopf.

Zusammenfassend besteht hohe Evidenz, dass die laparoskopische Resektion von Kolonkarzinomen onkologisch adäquat, sicher durchführbar und dem offenen Vorgehen gleichwertig ist. Das laparoskopische Vorgehen bei Patienten mit Kolonkarzinom sollte - bei besonnener Indikationsstellung und bestehender Expertise - das Verfahren der Wahl darstellen, um den Patienten die deutlichen Vorteile in der postoperativen Phase nicht vorzuenthalten. Dies sollte Eingang in die deutsche S3-Leitlinie finden. Als Beispiel kann hier die englische National Institute for Health and Clinical Excellence (NICE) Guideline dienen, die empfiehlt, Patienten laparoskopisch zu operieren, wenn sie für beide Verfahren geeignet wären.

\section{Disclosure Statement}

Es bestehen keine Interessenkonflikte.

\section{Literatur}

1 Hohenberger W, Weber K, Matzel K, Papadopoulos T, Merkel S: Standardized surgery for colonic cancer: complete mesocolic excision and central ligation - technical notes and outcome. Colorectal Dis 2009;11:354-364, discussion 364-365.

2 West NP, Hohenberger W, Weber K, Perrakis A, Finan PJ, Quirke P: Complete mesocolic excision with central vascular ligation produces an oncologically superior specimen compared with standard surgery for carcinoma of the colon. J Clin Oncol 2010;28:272-278.

3 Jacobs M, Verdeja JC, Goldstein HS: Minimally invasive colon resection (laparoscopic colectomy). Surg Laparosc Endosc 1991;1:144-150.

4 Hewett PJ, Allardyce RA, Bagshaw PF, et al: Short-term outcomes of the Australasian randomized clinical study comparing laparoscopic and conventional open surgical treatments for colon cancer: the ALCCaS trial. Ann Surg 2008;248:728 738.

5 Bagshaw PF, Allardyce RA, Frampton CM, et al: Long-term outcomes of the Australasian randomized clinical trial comparing laparoscopic and conventional open surgical treatments for colon cancer: the Australasian Laparoscopic Colon Cancer Study trial. Ann Surg 2012;256:915-919.

6 Lacy AM, García-Valdecasas JC, et al: Laparoscopy-assisted colectomy versus open colectomy for treatment of non-metastatic colon cancer: a randomised trial. Lancet 2002;359:2224-2229.

7 Lacy AM, Delgado S, Castells A, et al: The longterm results of a randomized clinical trial of laparoscopy-assisted versus open surgery for colon cancer. Ann Surg 2008;248:1-7.
8 Guillou PJ, Quirke P, Thorpe H, et al: Short-term endpoints of conventional versus laparoscopicassisted surgery in patients with colorectal cancer (MRC CLASICC trial): multicentre, randomised controlled trial. Lancet 2005;365:1718-1726.

$\checkmark$ Jayne DG, Guillou PJ, Thorpe H, et al: Randomized trial of laparoscopic-assisted resection of colorectal carcinoma: 3-year results of the UK MRC CLASICC Trial Group. J Clin Oncol 2007; 25:3061-3068.

10 Green BL, Marshall HC, Collinson F, et al: Longterm follow-up of the Medical Research Council CLASICC trial of conventional versus laparoscopically assisted resection in colorectal cancer. $\mathrm{Br} \mathrm{J}$ Surg 2013;100:75-82.

11 Veldkamp R, Kuhry E, Hop WCJ, et al: Laparoscopic surgery versus open surgery for colon cancer: short-term outcomes of a randomised trial. Lancet Oncol 2005;6:477-484.

12 Clinical Outcomes of Surgical Therapy Study Group: A comparison of laparoscopically assisted and open colectomy for colon cancer. N Engl J Med 2004;350:2050-2059.

13 Ohtani H, Tamamori Y, Arimoto Y, Nishiguchi Y, Maeda K, Hirakawa K: A meta-analysis of the short- and long-term results of randomized controlled trials that compared laparoscopy-assisted and open colectomy for colon cancer. J Cancer 2012;3: 49-57.

14 Jayne DG, Thorpe HC, Copeland J, Quirke P, Brown JM, Guillou PJ: Five-year follow-up of the Medical Research Council CLASICC trial of laparoscopically assisted versus open surgery for colorectal cancer. Br J Surg 2010;97:1638-1645.
15 Quirke P, Steele R, Monson J, et al: Effect of the plane of surgery achieved on local recurrence in patients with operable rectal cancer: a prospective study using data from the MRC CR07 and NCICCTG CO16 randomised clinical trial. Lancet 2009; 373:821-828.

16 Burns EM, Currie A, Bottle A, Aylin P, Darzi A, Faiz O: Minimal-access colorectal surgery is associated with fewer adhesion-related admissions than open surgery. Br J Surg 2013;100:152-159.

17 Casillas S, Delaney CP, Senagore AJ, Brady K, Fazio VW: Does conversion of a laparoscopic colectomy adversely affect patient outcome? Dis Colon Rectum 2004;47:1680-1685.

18 Rottoli M, Stocchi L, Geisler DP, Kiran RP: Laparoscopic colorectal resection for cancer: effects of conversion on long-term oncologic outcomes. Surg Endosc 2012;26:1971-1976.

19 Marusch F, Gastinger I, Schneider C, et al: Experience as a factor influencing the indications for laparoscopic colorectal surgery and the results. Surg Endosc 2001;15:116-120.

20 Laubert T, Bader FG, Kleemann M, et al: Outcome analysis of elderly patients undergoing laparoscopic resection rectopexy for rectal prolapse. Int J Colorectal Dis 2012;27:789-795.

21 Bader FG, Roblick UJ, Oevermann E, Bruch H-P, Schwandner O: Radical surgery for early colorectal cancer - anachronism or oncologic necessity? Int J Colorectal Dis 2008;23:401-407.

22 Bruch H-P, Esnaashari H, Schwandner O: Current status of laparoscopic therapy of colorectal cancer. Dig Dis 2005;23:127-134. 